Kahlert, Joachim

\title{
Sachunterrichtsplanung als begründungspflichtige Anforderung an professionelles Lehrerhandeln
}

Tänzer, Sandra [Hrsg.]; Lauterbach, Roland [Hrsg.]: Sachunterricht begründet planen. Bedingungen, Entscheidungen, Modelle. Bad Heilbrunn : Verlag Julius Klinkhardt 2010, S. 263-278

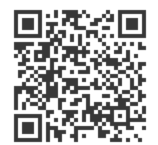

Quellenangabe/ Reference:

Kahlert, Joachim: Sachunterrichtsplanung als begründungspflichtige Anforderung an professionelles Lehrerhandeln - In: Tänzer, Sandra [Hrsg.]; Lauterbach, Roland [Hrsg.]: Sachunterricht begründet planen. Bedingungen, Entscheidungen, Modelle. Bad Heilbrunn : Verlag Julius Klinkhardt 2010, S. 263-278 - URN: urn:nbn:de:0111-pedocs-209329 - DOI: 10.25656/01:20932

https://nbn-resolving.org/urn:nbn:de:0111-pedocs-209329

https://doi.org/10.25656/01:20932

in Kooperation mit / in cooperation with:

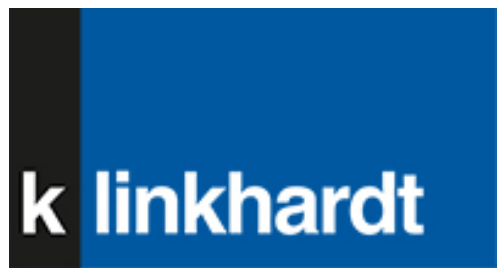

http://www.klinkhardt.de

\section{Nutzungsbedingungen}

Dieses Dokument steht unter folgender Creative Commons-Lizenz: http://creativecommons.org/licenses/by-nc-nd/4.0/deed.de - Sie dürfen das Werk bzw. den Inhalt unter folgenden Bedingungen vervielfältigen, verbreiten und öffentlich zugänglich machen: Sie müssen den Namen des Autors/Rechteinhabers in der von ihm festgelegten Weise nennen. Dieses Werk bzw. dieser Inhalt darf nicht für kommerzielle Zwecke verwendet werden und es darf nicht bearbeitet, abgewandelt oder in anderer Weise verändert werden.

Mit der Verwendung dieses Dokuments erkennen Sie die Nutzungsbedingungen an.

\section{Terms of use}

This document is published under following Creative Commons-License: http://creativecommons.org/licenses/by-nc-nd/4.0/deed.en - You may copy, distribute and transmit, adapt or exhibit the work in the public as long as you attribute the work in the manner specified by the author or licensor. You are not allowed to make commercial use of the work or its contents. You are not allowed to alter, transform, or change this work in any other way.

By using this particular document, you accept the above-stated conditions of use.

\section{Kontakt / Contact:}

\section{peDOCs}

DIPF | Leibniz-Institut für Bildungsforschung und Bildungsinformation Informationszentrum (IZ) Bildung

E-Mail: pedocs@dipf.de

Internet: www.pedocs.de

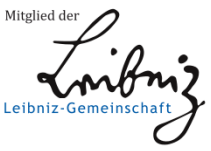




\title{
5.6 Sachunterrichtsplanung als begründungs- pflichtige Anforderung an professionelles Lehrerhandeln
}

\author{
von Joachim Kahlert
}

Ja, mach nur einen Plan!

Sei nur ein großes Licht!

Und mach dann noch 'nen zweiten Plan!

Gehn tun sie beide nicht.

(Bert Brecht, Ballade von der Unzulänglichkeit)

\subsubsection{Planung - keine Garantie für Gelingen, sondern Rechtfertigung für Vorhaben}

In der oben zitierten Ballade ironisiert Brecht eine Alltagserfahrung, die seit Langem auch Philosophen und Sozialwissenschaftler beschäftigt: Eigensinn und Subjektivität von Interaktionspartnern begrenzen die Planbarkeit sozialen Handelns (vgl. z.B. Gadamer 1967, 9; Luhmann 1997a, 496f.; ders. 1997b, 776ff.; Simmel 1906, 158). Das ist beim Unterrichten nicht anders.

Unterricht ist ein soziales Geschehen zwischen Subjekten, die ihre eigenen Interpretationen über das haben, was situativ gerade wichtig, interessant und erforderlich ist (vgl. Meyer 2008, 117-121). Dieses Geschehen ist nur begrenzt vorhersehbar und nicht bis ins Detail im Voraus planbar. Einzelne Schülerinnen und Schüler verhalten sich anders als erwartet. Die ausgewählten Aufgaben sind für die Schüler schwieriger als gedacht. Der Zeitplan kommt durcheinander. Selbst dann, wenn alles scheinbar reibungslos läuft, ist nicht gewiss, dass die Effekte des Unterrichts hinreichend den Absichten und Lernzielen entsprechen, die der Planung zugrunde lagen. Denn auch für Unterricht gilt Eduard Sprangers Bemerkung, dass „bei unserem Handeln noch immer etwas anderes herauskommt, als wir gewollt haben“" (Spranger 1962, 8). Die Anmerkungen zu den Grenzen der Planbarkeit sprechen nicht gegen sorgfältige Planung, sondern gegen sozial-technisch überzogene Erwartungen an die Planung. Lehrende, die ihren Unterricht bereits deshalb für erfolgreich halten, weil er „plangerecht“ verlief, verfallen dem Trugschluss einer zu engen Kopplung von Planung des Unterrichts und dem realen Geschehen im Unterricht genauso wie Beobachter des Unterrichts, die unerwünschtes Geschehen, Abweichungen von der geplanten Zeit, unerwartete Schwierigkeiten 
der Schülerinnen und Schüler kurzschlüssig auf Fehler in der Planung zurückführen. Hinterher ist man immer schlauer, doch als Beurteilter kann man nie beweisen, dass der konkrete Vorfall nicht vorhersehbar war. So wird Unvorhersehbares als Fehler (in der Planung) zurechenbar. In hierarchisch gestützten Abhängigkeitsverhältnissen, wie sie zum Beispiel zwischen Schulleitungen sowie Schulräten und den Lehrkräften bestehen oder auch zwischen Ausbildern der 2. Phase und den Referendaren, wirkt das entmündigend.

Die kritische Haltung gegenüber einem sozial-technischen Planungsverständnis rechtfertigt jedoch keineswegs mangelnde Sorgfalt bei der Planung. Ohne begründbare Vorstellungen von Zielen des Unterrichts und von Mitteln, mit denen sie erreicht werden könnten, ist keine aufbauende Bildungsentwicklung denkbar. In jeder Aktivität, und sei sie auch noch so spontan, realisiert sich eine Entscheidung. Die Aktivität schafft Differenz, man hat es so und nicht anders gemacht. Auch Unterrichten ist eine Abfolge von Entscheidungen (vgl. Kiel 2008, 21f.). Im Leben sonst ist es den einzelnen Akteuren überlassen, ob sie ihre Entscheidungen durchdacht haben und begründen können. Aber Entscheidungen, die man als Lehrer trifft, sind begründungspflichtig, und zwar nicht in erster Linie deshalb, weil vorgesetzte Instanzen, Eltern oder Schüler kritisch nachfragen könnten, sondern vor allem, weil die fachlich und pädagogisch nachvollziehbare Begründung für Entscheidungen ein Kennzeichen für professionelles Handeln im Lehrerberuf ist (vgl. Terhart 2005, 135f.). Es mag ja sein, dass es außerschulischen Experten, Künstlern oder begnadeten Entertainern gelegentlich gelingt, mit ihren speziellen Fähigkeiten Schulklassen oder Schülergruppen in den Bann zu ziehen. Aber es ist ein Unterschied, ob man Schülerinnen und Schüler mit partikularen, dafür aber hoch spezialisierten Fähigkeiten über einen kurzen Zeitraum begeistert oder ob man sie in einem auf viele Jahre angelegten Bildungs- und Erziehungsweg unter den Bedingungen schulischer und unterrichtlicher Zwänge nach bestem fachlichen und pädagogischen Wissen und Gewissen begleitet. Die Fähigkeit, auf hohem fachlichen und pädagogischen Niveau begründen und einordnen zu können, wie man in Schule und Unterricht handelt, unterscheidet den in Studium und Referendariat ausgebildeten professionellen Lehrer von Schmalspurlehrkräften, die Gelegenheitserfolge verbuchen.

Planung ist keine Erfolgsgarantie, sondern eine Art professionell legitimierte Hypothesenbildung: So könnte es gelingen, diese und jene Begründung stützen die Annahmen. Das Unterrichtsgeschehen selbst liefert die Daten, besser Indizien, für den pragmatischen Test dieser Hypothese, der mit der abschlieBenden Reflexion des Geschehens an Aussagekraft gewinnt. 
Gerade weil nicht alle Entscheidungen, die im realen Geschehen getroffen werden müssen, im Voraus bekannt sind, sollte das jeweilige Vorhaben gut begründet sein. Unerwartetes wird damit zwar nicht verhindert - und das wäre ja auch in einem pädagogischen Geschehen, das die Selbstständigkeit der Lernenden fördern sollte, nicht erstrebenswert. Aber das Nachdenken über die Gründe für ein geplantes Vorgehen mobilisiert Wissens- und Ideenressourcen, die dazu beitragen können, unerwartete Herausforderungen angemessen zu bewältigen. Und nach der Handlung erleichtert der Vergleich zwischen dem, was mit guten Gründen geplant war, und dem, was geschehen ist, die systematische Reflexion über den eigenen Unterricht (vgl. Wiater 2006a, 706f.).

Zu Recht verlangen daher Standards für die Lehrerbildung: „Lehrerinnen und Lehrer planen Unterricht fach- und sachgerecht und führen ihn sachlich und fachlich korrekt durch" (Sekretariat der Ständigen Konferenz der Kultusminister, 7; siehe auch Oser 2001, 236). Schaut man in die Literatur, die sich mit Fragen der Unterrichtsplanung näher beschäftigt, dann wird man von der Vielfalt der zu begründenden Entscheidungen schier überwältigt. Lernstand, Entwicklungspotenziale, Erfahrungen und Interessen der Schülerinnen und Schüler sollen angemessen berücksichtigt, Lernziele mit Bezug auf übergreifende Bildungsansprüche formuliert und begründet, der Unterrichtsablauf nach neuesten Kenntnissen der Lehr-Lernforschung gestaltet werden. $\mathrm{Zu}$ bedenken seien außerdem mögliche Lernschwierigkeiten, Differenzierungen, Indikatoren für den Lernerfolg, Maßnahmen zur Sicherung des Lernertrages, einzusetzende Medien und die fachlichen Anforderungen der Unterrichtsinhalte. Schließlich soll das Vorhaben zum Lehrplan passen und Überlegungen zum weiterführenden Lernen berücksichtigen (vgl. z.B. Sandfuchs 2006; Wiater 2006b;). Hinzu kommen die spezifischen Anforderungen des jeweiligen Unterrichtsfaches - wie zum Beispiel des Sachunterrichts.

Wer (Sach)unterricht plant, hält und analysiert, trifft Entscheidungen unter anderem über Inhalte, Methoden, Medien, Zeiteinteilung. Wie lässt sich das Vorwissen der Schülerinnen und Schüler zu dem Unterrichtsinhalt aktivieren? Eignet sich das Thema für eine problemorientierte Bearbeitung? Ist ein Heimatbezug sinnvoll? Welche Erkenntnisse über kognitive, emotionale und praktische Fähigkeiten und Fertigkeiten, die im Grundschulalter wahrscheinlich sind, können im Zusammenhang mit dem Unterrichtsinhalt herangezogen werden? Entscheidungen über diese und viele weitere Fragen müssen vor dem Hintergrund professioneller Standards, wie zum Beispiel grundlegender Bildungs- und Erziehungsziele, anerkannten fachdidaktischen und (grund-) schulpädagogischen Wissens, Theorien über schulisches Lehren und Lernen und gültiger Lehrpläne, begründbar sein. Dabei sind auch eine Vielzahl situativer Bedingungen einzubeziehen, die von Lerngruppe zu Lerngruppe und 
von Schule zu Schule variieren: Lern- und Leistungsvoraussetzungen der jeweiligen Schülerinnen und Schüler, räumliche Gegebenheiten und Sachausstattung in der Schule, zeitliche Zwänge. Nicht zuletzt müssen die jeweils getroffenen Entscheidungen sich auch mit Bezug auf das eigene professionelle Profil im Hinblick auf Methodenvielfalt, Ideenreichtum, Umgang mit Schülerinnen und Schülern, fachliche Kenntnisse und vieles mehr rechtfertigen lassen. Nähme man sich vor, jede dieser Entscheidungen im Einzelnen zu begründen, dann würde man sich in Detailfragen verlieren. Ein eleganter Handlungsplan für Unterricht entsteht jedoch nicht wie ein Puzzle, das aus zahlreichen Einzelteilen zusammensetzbar ist, sondern eher wie ein Drehbuch: Ausgehend von einer Leitidee (dem „Plot“ bzw. dem mehr oder weniger präzise umrissenen Unterrichtsinhalt, der zum Thema wird) wird über Ziele, mögliche Vorgehensweisen, verfügbare Ressourcen, Eigenheiten der Lernenden und vieles mehr nachgedacht. Nach und nach gewinnt die Idee die Gestalt eines Handlungsplans. Dabei beeinflussen sich einzelne Überlegungen und man kann zahlreiche Freiräume für kreative und überraschende Ideen nutzen. Trotz dieser Vielfalt in der Such- und Entwurfsphase lässt sich eine Grundstruktur für begründungspflichtige Entscheidungen identifizieren. Schulischer Unterricht beansprucht für einen vorgegebenen Zeitraum die Lebenszeit junger Menschen im öffentlichen Auftrag und mit öffentlichen Mitteln. ${ }^{1}$ Damit stellen sich drei grundlegende Fragen:

(a) Wie rechtfertigt man angesichts knapper Zeit und knapper Mittel, dass gerade der ausgewählte Inhalt zum Unterrichtsthema wird?

(b) Lassen sich die Wirkungserwartungen, die mit dem Unterrichtsinhalt verbunden sind, mit Bezug auf die Lernvoraussetzungen der Schülerinnen und Schüler und unter Berücksichtigung der fachlichen Anforderungen begründen?

(c) Wie begründet sich das für den jeweiligen Zeitraum geplante Lernarrangement unter Berücksichtigung der Rahmenbedingungen in der jeweiligen Schule?

Diese drei Entscheidungsfelder hängen eng miteinander zusammen. Wie man im Unterricht vorgeht (c), ist (hoffentlich) auch vom Wissen über den Kenntnisstand der Schülerinnen und Schüler (b) und von grundlegenden Bildungszielen abhängig (a). Diese wiederum wird man nicht sinnvoll konkretisieren können, ohne die Lernvoraussetzungen der Schüler und die fachlichen Anforderungen der Sache (b) mit zu denken.

Obwohl Entscheidungen zu unterschiedlichen Bereichen zusammenspielen, stützen sich die Begründungen für die Entscheidungen jeweils auf unter-

1 Dass dieses ersatzweise auch in privater Trägerschaft geschehen kann, ändert nichts an der Pflicht des Schülers, am Schulunterricht teilzunehmen und an der Pflicht des Staates zu prüfen, ob Angebote und Rahmenbedingungen dem Bildungsauftrag gerecht werden. 
scheidbare Theorie- und Wissensgebiete. So beziehen sich Begründungen für die Auswahl von Inhalten (a) auf bildungstheoretisch reflektierte Argumente. Wirkungserwartungen (b) müssen mit Bezug auf erziehungswissenschaftlich relevante Kenntnisse und fachliches Hintergrundwissen gerechtfertigt werden. Und schließlich müssen sich die Begründungen für das geplante Lernarrangement (c) sowohl auf Theorien und Erkenntnisse über Lehren und Lernen (im Unterricht) als auch auf die konkreten Rahmenbedingungen in der jeweiligen Schule beziehen.

Im Folgenden soll zunächst jedes dieser Entscheidungsfelder näher beleuchtet werden (Teil 2). Danach wird an einem Beispiel konkretisiert, wie Entscheidungen in diesen drei Feldern zusammenwirken können (Teil 3).

\subsubsection{Begründungspflichtige Dimensionen der Sachunterrichtsplanung}

\section{Die bildungstheoretische Dimension: Was wird zum Thema - und warum?}

Lebenszeit ist begrenzt, Unterrichtszeit noch stärker. Sachunterricht soll Kinder dabei unterstützen, ihre Umwelt mit Bezug auf zuverlässiges Wissen zu erschließen und Grundlagen für Sachfächer weiterführender Schulen legen. Da die Umwelt weitaus mehr Lernanlässe bietet als in der auf wenige Schuljahre und Unterrichtsstunden begrenzten Zeit jemals zu bewältigen ist, stellt sich die grundlegende Frage: Welche Inhalte und Themen verdienen es, im Unterricht zur Sprache zu kommen? ${ }^{2}$

Zwar trifft der Lehrplan eine Vorentscheidung, aber er determiniert die Inhalte nicht und er lässt Spielraum für Schwerpunktsetzungen. Außerdem kann niemand einen Inhalt ergiebig unterrichten, ohne sich Rechenschaft über die kulturelle und pädagogische Bedeutung des Inhalts zu geben. Gerade dann, wenn - wie in guten Lehrplänen - viele starke Angebote miteinander konkurrieren, muss das, was zum Inhalt wird, didaktisch gut begründet werden. Der kulturelle Stellenwert des Unterrichtsinhalts erschließt sich mit Antworten auf die Leitfrage, welche Bedeutung es für das Zusammenleben heute und in Zukunft hat, wenn Kinder in der Schule diesem Inhalt begegnen. Warum ist es mit Blick auf die Gesellschaft sinnvoll, dass junge Menschen etwas über Heimatgeschichte, den Stromkreis, die einheimischen Tiere und Pflanzen erfahren? Unter pädagogischem Blickwinkel lautet die Leitfrage: Warum ist dies sinnvoll für die Persönlichkeitsentwicklung des einzelnen Kindes? Didaktische Entscheidungen, also die Auswahl und Begründung von Lerninhalten, beeinflussen, womit Schülerinnen und Schüler kostbare Lebenszeit

\footnotetext{
2 Warum die Unterscheidung von Inhalt und Thema gerade in der Sachunterrichtsdidaktik wichtig ist, wird in Kahlert 2009, 205f. ausgeführt.
} 
verbringen und berühren zentrale Probleme des gesellschaftlichen Zusammenlebens in modernen Gesellschaften. Wenn man es nicht dem Zufall von Geburt, Herkunft und Lebensweisen überlassen möchte, welche Chancen Kinder haben, etwas Sinnvolles über diese Welt zu erfahren, dann muss sich eine Institution dafür verantwortlich fühlen und diese schwierige, aber für das zivilisierte Zusammenleben so unverzichtbare und wertvolle Aufgabe übernehmen - und das ist die Schule. Ihr kommt, sowohl mit Blick auf die Persönlichkeitsentwicklung des Einzelnen als auch mit Blick auf das Zusammenleben mit anderen, die grundlegende Aufgabe zu, allen Schülerinnen und Schülern angemessene Lernchancen zu bieten. Unabhängig von den Besonderheiten ihrer Lebenswelt sollen Kinder und Jugendliche diejenigen Fähigkeiten, Kenntnisse und Fertigkeiten erwerben können, die nach bestem pädagogischen Wissen und Gewissen ihrer persönlichen Entwicklung und dem Zusammenleben mit anderen dienen. Bezogen auf natur- und sozialwissenschaftlich deutbare Phänomene, Gegebenheiten, Entwicklungen und Beziehungen ist der Sachunterricht in der Grundschule der Lernbereich, in dem dafür Grundlagen gelegt werden können. Er sorgt mit dafür, dass alle Kinder gleiche Chancen haben, aus natur- und sozialwissenschaftlichen Gebieten etwas zu lernen, was sinnvoll zu begründen ist - und zwar unabhängig davon, ob die Kinder in Flensburg oder in Starnberg, in Trier oder in Bautzen aufwachsen, in reichen oder armen Familien, katholisch, evangelisch, muslimisch oder atheistisch. Damit wirkt Sachunterricht daran mit, eine Basis für die Verständigung über die Grenzen von Gender, Kultur, Religionen und Milieu hinweg zu sichern. In diesem Sinne kann man ihn auch als das Kerncurriculum für Demokratie und Kultur verstehen. Didaktische Entscheidungen im Sachunterricht sind daher Entscheidungen von hohem Rang; sie sollten entsprechend fundiert begründet werden können.

Dabei geht es nicht darum darzulegen, die Auswahlentscheidung für einen Inhalt sei ,richtig“. Dies würde einen argumentativen Determinismus voraussetzen, für den es schon allein auf Grund der Vielfalt miteinander konkurrierender Theorien über gegenwärtige und zukünftige Herausforderungen des Zusammenlebens keine vernünftige Basis gibt. Ziel der didaktischen Begründung ist es vielmehr, die jeder Auswahlentscheidung anhaftenden impliziten Vorstellungen über Gegenwart und Zukunft explizit und somit verhandelbar zu machen und dabei auch die jeweils vorherrschenden übergreifenden Bildungs- und Erziehungsziele zu berücksichtigen.

Wenn man die (sachunterrichts-)didaktische Literatur der letzten Jahrzehnte durchblättert, dann trifft man auf ein breites Spektrum solcher Leitziele: Heimatliebe, Förderung des wissenschaftlichen Denkens, Emanzipation, Bewältigung von Anforderungen des Alltags, Befähigung zur demokratischen Teilhabe und vieles mehr. In neuerer Zeit besteht der Anspruch, Ziel- 
setzungen als Kompetenzen zu formulieren (vgl. Giest u.a. 2008). Es gibt zwar zahlreiche Definitionen für Kompetenz, aber die für pädagogische Anwendung brauchbaren Vorstellungen von Kompetenz betonen recht einheitlich, damit sei ein Zusammenspiel von Fähigkeiten und Fertigkeiten gemeint, das es erlaubt, mehr oder weniger klar definierten Anforderungen für Handlungssituationen gerecht zu werden. Kompetenzen sind erlernbar und gründen auf das Zusammenwirken von Wissen, Einstellungen, Motiven, Werten und Erfahrungen, Fähigkeiten und Fertigkeiten. Sie beschreiben damit das Vermögen, Anforderungen in einem komplexen Aufgabengebiet zu bewältigen.

Wir können an dieser Stelle keinen Katalog der Kompetenzen vorstellen, die im Sachunterricht anzustreben wären. Doch wenn man Kompetenzen als Zusammenspiel von Wissen, Können und Verstehen begreift, dann sollten Inhalte und Themen auch danach befragt werden, was sie leisten, um das Verständnis der Kinder über ihre Umwelt zu fördern und um das Vermögen der Kinder zu erweitern, in der Sache verständig und gegenüber sich selbst und ihrer Umwelt verantwortungsvoll zu handeln. Zusammenfassend lassen sich daher folgende Fragen formulieren, die hilfreich sind, um die Auswahl von Inhalten und Themen des Sachunterrichts fundiert zu begründen:

○ Inwieweit ist der Inhalt für die Bewältigung lebensweltlicher Anforderungen heute und in Zukunft bedeutsam?

- Warum ist es für das Zusammenleben sinnvoll, wenn Kinder etwas darüber lernen?

- Welche Argumente sprechen dafür, dass dies die Persönlichkeitsentwicklung des einzelnen Kindes fördern kann?

- Was sollten Kinder darüber wissen und verstehen?

$\circ \quad$ Welche kurz- und mittelfristigen Könnensziele sind mit dem Inhalt verbunden?

In dem Maße, wie Antworten auf diese Fragen gefunden werden, klärt sich der Bildungsgehalt der Inhalte und Themen. Ob er sich auch realisieren lässt, hängt wiederum davon $a b$, ob man sich als Unterrichtender hinreichend treffende Vorstellungen über lernrelevante Voraussetzungen der Schülerinnen und Schüler und über die sachlichen bzw. fachlichen Anforderungen macht, die mit dem Unterrichtsinhalt verbunden sind.

\section{Wirkungserwartungen begründen: Den Lerner im Blick - und die Sache im Kopf}

Es gehört heute zu den als gültig angenommenen Erkenntnissen der Unterrichtsforschung, dass lernwirksamer Unterricht sich durch Klarheit und Systematik in der Sache und durch Adaptivität in Bezug auf die Schüler auszeichnet (vgl. Helmke 2009, 191ff. und 246ff.; Meyer 2004). Die Erwartung, Unterricht möge im Sinne der zugrunde liegenden Absichten wirken, muss 
sich damit sowohl im Hinblick auf die Lernvoraussetzungen der Kinder als auch auf die fachlichen Herausforderungen des Unterrichtsinhalts rechtfertigen lassen. An anderer Stelle wurde ausführlich dargelegt, wie es im Sachunterricht gelingen kann, den Lernvoraussetzungen der Kinder und den Ansprüchen der Sache gerecht zu werden (vgl. Kahlert 2009, 216ff.). Selbstverständlich wird man nie alle relevanten Kenntnisse und Informationen über die Kinder einholen können. Hier sollen die Perspektiven begründet werden, unter denen man planungsrelevante und belastbare Vorstellungen über die Lernvoraussetzungen der Kinder gewinnt. Die entwicklungsorientierte Perspektive bietet Begründungen für Wirkungserwartungen mit Blick auf kognitive, emotionale und soziale Fähigkeiten, mit denen in einem bestimmten Alter zu rechnen ist. Welches Niveau an Differenzierung und Integration von Umweltwahrnehmungen ist wahrscheinlich? Mit welchen Gedächtnisleistungen kann man rechnen? Sind die Kinder bereits in der Lage, die Perspektive eines anderen einzunehmen und im eigenen Handeln zu berücksichtigen? Unter einer sozio-kulturell orientierten Perspektive kommen als Begründung Merkmale in Betracht, die für das Alltagsleben der Kinder von Bedeutung sind: Verbreitete Gewohnheiten, typische Erfahrungen, vorherrschende Interessen, aber auch unterschiedliche Chancen von Kindern, an den gesellschaftlich zur Verfügung stehenden Lern- und Entwicklungsmöglichkeiten teilzuhaben sowie Anforderungen, die das moderne Leben an die Kinder stellt. Schließlich ist unter einer anthropologischen Perspektive pädagogisches Handeln mit Bezug auf ein Menschenbild zu begründen. Diese Perspektive bietet eine Grundlage für normativ begründete Bildungs- und Erziehungsziele sowie für normativ begründete Anforderungen an die Gestaltung von Lernund Entwicklungsumwelten. Damit nimmt sie der pädagogischen Praxis den Schein von Selbstverständlichkeiten. Die Verantwortung für pädagogische Entscheidungen verschwimmt, wenn Einflussnahmen auf das Leben eines anderen Menschen mit nicht hinterfragten Ansprüchen über die Gestaltung des Zusammenlebens begründet werden. Mit der Frage nach dem Menschenbild, das sich in pädagogischen Handlungen realisiert, wird es notwendig, Standpunkt zu beziehen und Verantwortung zu übernehmen.

Die drei Bezugssysteme für Begründungen ergänzen sich und können sich gegenseitig kontrollieren. Die sozio-kulturell argumentierende Begründung wirkt der Gefahr entgegen, dass man verbreitete Eigenarten und Merkmale für grundlegende anthropologische Gegebenheiten hält, obwohl sie kulturell erworben sind. Ein Beispiel dafür sind Annahmen über die Bedürfnisse von Menschen oder viele geschlechtsspezifische Zuschreibungen. Anthropologische Bezüge wiederum helfen, die Auswirkungen sozio-kultureller Entwicklungen auf Kinder nicht lediglich hinzunehmen, sondern auch vor dem Hintergrund von Menschenbildern zu bewerten. Und schließlich grenzt die Be- 
gründung von Entscheidungen mit entwicklungsorientierten Argumenten die Gefahr ein, an dem Vorstellungs- und Verarbeitungsvermögen der Lernenden vorbei zu planen und zu unterrichten. Weder ist der sozio-kulturelle Hintergrund völlig erschließbar, noch lassen sich alle relevanten entwicklungsbezogenen Informationen über die Kinder ermitteln. Sicherheit gibt es auch hier nicht. Der begründende Bezug auf die Lernvoraussetzungen schafft deshalb auch keine Gewissheit über die Angemessenheit der Planung. Vielmehr leistet der Bezug auf die Lernvoraussetzungen nicht mehr - aber auch nicht weniger - als die Frage zu klären, wie sich Wirkungserwartungen mit Bezug auf Interessen, Erfahrungen und Fähigkeiten und dabei gestützt auf anerkanntes Wissen und akzeptierte Theorien begründen lassen.

Die fachliche Absicherung von Annahmen über die Lernvoraussetzungen der Schüler ist nur die eine Seite des Versuchs, Wirkungserwartungen zu begründen. Hinzukommen muss die Berücksichtung von Herausforderungen, die in der Sache liegen. So lassen sich zum Beispiel Phänomene des Luftdrucks nicht verstehen, ohne den Kraftbegriff ins Spiel zu bringen. Über den Wasserkreislauf lässt sich nichts Sinnvolles lernen, wenn nicht auch die verschiedenen Aggregatzustände des Wassers geklärt werden. Das demokratische Prinzip einer Klassensprecherwahl erschließt sich nicht, ohne über die Rechtfertigung von verliehener Macht bzw. Einflussnahme zu sprechen. Unter Sachgesichtspunkten ist daher zu begründen, wie die jeweilige Einzelstunde sachlich angemessen in eine größere Unterrichtseinheit eingepasst wird. Gibt es von der Sache her eine Aufbau- und Verlaufslogik, die zwingend zu berücksichtigen ist? Welche relevanten Begriffe sind zu klären? Sind die Sachverhalte fachlich korrekt erfasst und bieten sich Gelegenheiten, übergreifende Zusammenhänge zu anderen Sachbezügen zu thematisieren?

Angesichts seiner enormen inhaltlichen Breite, die von naturwissenschaftlichen Phänomenen über geographische, geschichtliche, wirtschaftliche bis hin zu sozialwissenschaftlichen Themengebieten reicht, stellt die fachliche Durchdringung von Sachverhalten eine große Herausforderung für die Lehrerinnen und Lehrer dar. Die Vorstellung, dies sei ja nicht so anspruchsvoll, weil man es ja nur mit Grundschulniveau zu tun habe, ist abwegig. Gerade die elementaren Einsichten und der heute geforderte Aufbau von Kompetenzen im Bildungsgang erfordern gründliches Verständnis von der Sache, die unterrichtet wird. Erst dieses Sachverständnis macht es möglich zu entscheiden, welche Einsichten für die Schülerinnen und Schüler wichtig sind, wo Schwierigkeiten in der Sache liegen und welche inhaltlichen Voraussetzungen zunächst vorhanden sein müssen, damit die angestrebten Einsichten auch tatsächlich erreicht werden können. Hinzu kommen lernbereichsspezifische fachdidaktische Anforderungen: Die das Lernen unterstützende Aktivierung von Vorwissen der Schülerinnen und Schüler erfordert beim Unterrichten 
von ökonomischen Inhalten andere Impulse als Unterricht zu physikalischen Themen. Bei der Einführung in das Kartenverständnis kommen andere Unterrichtsmethoden infrage als zur Entwicklung der Fähigkeit, kritisch mit Textund Bildquellen über geschichtliche Ereignisse umzugehen.

Die einer Unterrichtsplanung innewohnenden Wirkungserwartungen sollten somit in Bezug sowohl auf die begründet anzunehmenden Lernvoraussetzungen als auch auf die fachlichen Anforderungen gerechtfertigt werden können:

- An welches Vorwissen und an welche Erfahrungen und Interessen kann der ausgewählte Inhalt anschließen?

$\circ$ Gibt es in diesem Zusammenhang Herausforderungen, Orientierungsprobleme, typische Kinderfragen, an die der Unterricht anknüpfen könnte?

- Berücksichtigt die Planung hinreichend die sozialen, emotionalen und kognitiven Fähigkeiten der Kinder in Bezug auf den Unterrichtsgegenstand?

- Wird die Planung den fachlichen Anforderungen der Sache gerecht (z.B. in der Reihenfolge der zu behandelnden Sachverhalte)?

- Ist fachlich hinreichend erläutert, auf welche Einsicht es unter fachlichen Gesichtspunkten besonders ankommt?

\section{Lernarrangements begründen - der konkrete Ablauf}

Wenn die Ziele des Unterrichts und die Wirkungserwartungen hinreichend begründet sind, kommen als dritte Begründungsdimension für das geplante Geschehen Erkenntnisse und Theorien über wirksame Lehr-Lernsituationen unter Berücksichtigung der konkreten unterrichtlichen Bedingungen ins Spiel. Man mag von der Lehr-Lernforschung gelernt haben, dass Unterricht besonders wirksam ist, wenn er an motivierenden Problemen ansetzt, Schülererfahrungen stimuliert und Lösungsbeispiele, Übungssituationen sowie Anwendungsmöglichkeiten bietet. Wie diese grundlegenden Erkenntnisse wirksam werden, hängt jedoch von den besonderen Umständen des Unterrichts ab. In großen Gruppen werden andere Anwendungsübungen gewählt werden müssen als in kleinen Klassen. Stehen mehrere zusammenhängende Unterrichtsstunden zur Verfügung, können Erkundungen eher eingeplant werden als in einer 45-Minuten-Stunde. Die Verfügbarkeit von Medien setzt medienpädagogisch wünschenswerten Impulsen eine pragmatische Grenze. Nicht zuletzt spielen auch das eigene Können und die Erfahrungen der Lehrerinnen und Lehrer bei der Rechtfertigung der konkreten Ablaufplanung eine Rolle. Kataloge mit Ansprüchen an ,gute Lehrer“ gibt es viele. Aber selbst dann, wenn man unterstellt, alle Lehrerinnen und Lehrer wären bereit und in der Lage, sich erwünschte methodische und kommunikative Fähigkeiten anzueignen - im Moment der Planung und im Horizont einer baldigen Reali- 
sierung der Planung steht nur das zur Verfügung, was man bereits kann oder sich zutraut, nicht das, was man irgendwann in einer Fortbildung noch lernen wird. Wer noch wenig Erfahrung mit Stationenarbeit gesammelt hat, wird diese Arbeitsform nicht gerade bei Unterrichtsinhalten einsetzen, die auch fachlich eine besondere Herausforderung darstellen. Versucht man es doch, bedarf auch dies einer besonderen Begründung. Sicherlich kann und muss man sich erproben, aber auch hier gilt: auf der Stufe der sinnvollen nächsten Entwicklung. ${ }^{3}$

Unterricht kann und muss sich immer auch vor dem Hintergrund der konkreten Rahmenbedingungen rechtfertigen lassen. Diese sind so variantenreich, dass sie nur für jeden Einzelfall angemessen erfassbar sind und beurteilt werden können. Dennoch gibt es eine Grundstruktur des Unterrichtsgeschehens. Ob eine einzelne Stunde, eine Doppelstunde oder eine ganze Unterrichtseinheit geplant wird - immer steht nur ein begrenzter Zeitraum zur Verfügung, in dem sich Lernen ereignen soll. In diesem Zeitraum müssen die Schüler für das Thema gewonnen und ihnen muss Zeit gewährt werden, um Fragestellungen und Informationen zu erarbeiten, neues Wissen zu sichern, zu erproben und anzuwenden. In der Literatur gibt es dafür zahllose Strukturierungsvorschläge, die als Artikulationsschema des Unterrichts kommuniziert werden (vgl. z.B. Kiel 2008, 24ff.). Sturer Schematismus, wie er mitunter in Rezepturen für die Unterrichtsplanung zum Ausdruck kommt, ist dabei allein schon deshalb nicht sinnvoll, weil nicht in jeder zu planenden Zeiteinheit das ganze Spektrum der diversen Artikulationsschemata abgearbeitet werden muss, wie „Motivierender Einstieg“, „Fragestellung“, „Erarbeitung“, „Sicherung“, „Anwendung“ - und wie die Teilphasen auch immer heißen oder genannt werden mögen. Dennoch macht es die prinzipielle Begrenzung der zur Verfügung stehenden Zeit notwendig, Entscheidungen zur Dramaturgie des Unterrichts, also zur zeitlichen Taktung wesentlicher Elemente, zu treffen - und zu begründen.

So geht es zunächst überhaupt darum, die Lernenden für das Anliegen des Unterrichts zu gewinnen (vgl. Merrill 2002). Ob dies auf der Grundlage einer gemeinsamen Planung geschieht, durch Inszenierung eines interessanten Ereignisses, durch Konfrontation mit einem herausfordernden Problem, der Begegnung mit einer Schwierigkeit - entscheidend ist, dass mit der ersten Begegnung eine Erwartung entsteht. Es lohnt sich, aufmerksam zu sein, sich dem Gegenstand zuzuwenden. Klassisch formuliert: Der Einstieg sollte moti-

3 Nicht zuletzt auch deshalb ist eine intensive Reflexion und Begründung der Planung nötig: Sie hilft zu sehen, wo man als Lehrer steht und was man sich im Sinne der professionellen Weiterentwicklung noch für das eigene Lernen vornehmen sollte. 
vierend sein und das Interesse der Schülerinnen und Schüler wecken oder stimulieren. Auch wenn diese erste Begegnung eindrucksvoll arrangiert ist, mündet sie nicht zwangsläufig in ein Lernanliegen. Die Schüler müssen für eine Auseinandersetzung mit dem Unterrichtsgegenstand gewonnen werden, die über die erste Motivation hinausreicht. Dies kann ein Handlungsziel, ein Könnensziel oder auch das Interesse sein, eine Irritation aufzuklären, unterschiedliche Meinungen zu klären oder zu vergleichen. Ferner müssen in geeigneten Arbeitsschritten Informationen erarbeitet, festgehalten, in einen Zusammenhang mit dem Ausgangsproblem gebracht und zur Lösung des ursprünglichen Anliegens verarbeitet werden. Ob die dafür notwendigen Arbeitsschritte problemorientiert mit den Kindern entwickelt oder eher vom Lehrer gelenkt vorgegeben werden - in jedem Fall sollte den Kindern deutlich werden, was zu tun ist, um das anfängliche Interesse sinnvoll aufzugreifen. Schließlich sollte zum Abschluss der Beschäftigung mit einem Anliegen ein Ergebnis vorliegen, das von den Schülerinnen und Schülern in einen sinnvollen Zusammenhang mit ihrem Anliegen und mit der Bearbeitung dieses Anliegens gebracht werden kann. Dieses Ergebnis muss nicht unbedingt ein greifbares Handlungsprodukt sein. Auch eine neue Fähigkeit, eine gewonnene Einsicht, neues Wissen, neue Gesichtspunkte, ja selbst ein differenzierteres Verständnis von einem Problem kann dazu führen, dass der Lernprozess von den Schülerinnen und Schülern als sinnvoll erfahren wird.

Es wäre eine Überbürokratisierung, diese vier Kernelemente einer Unterrichtsdramaturgie - motivierender Anfang, einsichtige Arbeitsperspektive, konzentrierte Erarbeitung, sinnvoller Abschluss - zu einem starren Ablaufschema für Unterricht, zum Beispiel im Sinne hintereinander einzuhaltender Formalstufen, zu verknüpfen. Vielmehr sind sie als Aufgaben für die Unterrichtsplanung und als Gütekriterien zur Beurteilung einer Planung zu verstehen. Während der Planung dienen sie dazu, die Qualität des Vorhabens zu sichern. Nach Abschluss der Planung, und bevor sie in reales Geschehen mündet, ermöglichen sie im Sinne eines „Checks“ eine nochmalige Qualitätskontrolle. Und nach dem Unterricht können sie die gezielte Reflexion über den Unterricht strukturieren. Daher sollte sich die Begründung einer Unterrichtsplanung auch auf folgende Fragen beziehen:

- Ist der Unterrichtsgegenstand hinreichend motivierend eingeführt worden?

- Haben die Schüler Gelegenheit gehabt, ein für sie bedeutsames Anliegen mit dem Unterricht zu entwickeln?

- Sind die im Zusammenhang mit dem Unterrichtsthema auszuführenden Schülertätigkeiten den Lernenden hinreichend verständlich geworden, das heißt, wissen sie, was sie tun sollen und vor allem, warum sie das im Zusammenhang mit ihrem Interesse am Thema tun? 
- Wird das Ergebnis der Bemühungen festgehalten und reflektiert? Haben die Schüler die Gelegenheit, ihren Wissens- und Könnenszuwachs zu erfahren?

\subsubsection{Wie Begründungen Konturen schaffen - ein Beispiel}

Unterrichtsplanung ist ein theorie- und wissensgeleiteter kreativer Akt. Gelingt sie, wirkt das Produkt gut komponiert. Alle Teile sind in sich und im Zusammenspiel möglichst stimmig und bilden ein überzeugend begründbares Gesamtwerk. Dieses entsteht nicht durch bloße Anwendung von Wissen, zum Beispiel über den Lernprozess oder über die Lernvoraussetzungen der Schüler, sondern eher im Prozess eines aufgeklärten Experimentierens mit Ideen, die mit Bezug auf fachliches und pädagogisches Wissen ausgearbeitet und begründet werden und dabei nach und nach die Gestalt eines Handlungsplans annehmen. So entspringt bereits die erste Unterrichtsidee nicht, quasi als letzter Akt des Nachdenkens, aus dem erworbenen Wissen über Sachverhalte, Lernvoraussetzungen und Lernen. Das wäre eine mechanistische und auch verzweifelte Vorstellung über die Planbarkeit von Ideen. Vielmehr kann sie aus ganz unterschiedlichen Quellen stammen: der Routine, einer Anregung aus dem Lehrplan, einer zufälligen Beobachtung, einem Bild, einer Schüleräußerung, einem Buch. Im Planungsprozess selbst und für eine gegebenenfalls erforderliche schriftliche Ausarbeitung der Planung wird die erste Idee mehrfach bearbeitet, auf ihre Tragfähigkeit hin untersucht, im Lichte der in Teil 2 dargelegten Fragen geprüft, verworfen, umgekrempelt - und immer belastbarer ausgearbeitet. Ideen und die Suche nach einer Begründung konstituieren somit eine Feedback-Spirale, in der die Ideen immer präziser und die Begründungen immer überzeugender werden. Im vorliegenden Beitrag kann dieser Prozess nicht an Hand einer ausführlich ausgearbeiteten Unterrichtsplanung veranschaulicht werden (vgl. dazu Kahlert 2009, 249-257). Aber zumindest lassen sich die ersten Schritte von einer grundlegenden Idee hin zu einer groben Strukturierung einer Unterrichtseinheit an einem Beispiel konkretisieren. Dazu wähle ich den ökonomischen Lernbereich des Sachunterrichts.

Die Verschuldungskrise und einige Presseartikel über den Mangel an ökonomischem Wissen geben den Impuls, den Unterrichtsinhalt „Taschengeld“, bisher routiniert in zwei Stunden abgehandelt, in eine Unterrichtseinheit über Geld einzubetten. Thematisiert werden soll, wie Geld funktioniert, wie Bedürfnisse und Wünsche zustande kommen, wie man haushälterisch geschickt mit Geld umgeht und was man tun könnte, wenn man zu wenig Geld hat, um ein Bedürfnis zu befriedigen, aber auch, was man eher unterlassen sollte (leichtfertiges Verschulden). 
Begründen lässt sich dieses Vorhaben mit dem Stellenwert, den ein reflektierter Umgang mit Geld für die Selbständigkeit der Kinder heute und in Zukunft hat. Kinder im Grundschulalter kommen in ihrem Alltag mit wirtschaftlichen Fragen und Werten in Berührung (sozio-kulturelle Perspektive). Sie erhalten Taschengeld, bekommen Geldgeschenke zum Sparen und haben an den finanziellen Plänen und Sorgen der Familie teil. Konsumwünsche und -entscheidungen, Verschuldung oder gar Armut und Arbeitslosigkeit in der Familie gehören für viele Kinder zur aktuellen Lebenswirklichkeit. Recherchiert man, angestoßen durch diese noch sehr naheliegende Überlegung, etwas weiter, dann stößt man vielleicht auf folgende Information im Internet: Laut der Kids-Verbraucheranalyse 2009 (Egmont Ehapa Verlag 2009), einer jährlichen Studie zum Verbraucher- und Medienverhalten junger Zielgruppen, verfügen die 5,7 Millionen 6- bis 13-Jährigen trotz Wirtschaftskrise in diesem Jahr über insgesamt rund sechs Milliarden Euro. Rund 3,6 Milliarden Euro davon befinden sich der Studie zufolge auf Sparkonten, das sind durchschnittlich 626 Euro pro Kind. Fast 2,5 Milliarden Euro kommen durch Geldgeschenke und Taschengeld zusammen. Der Umgang mit eigenen Wünschen und Bedürfnissen ist eine dem menschlichen Dasein aufgegebene Herausforderung, die mit der Bedürftigkeit des Menschen und der besonderen Abhängigkeit der Kinder von umsorgenden Erwachsenen zusammenhängt (anthropologische Perspektive). Schließlich erhellt die entwicklungsorientierte Perspektive, dass sich erste Vorstellungen über Eigentum, Nutzungsrechte und über den sorgsamen Umgang mit dem Eigentum anderer ausgebildet haben. Allerdings haben Kinder im Grundschulalter noch keine relational belastbare Größenvorstellung von Geldsummen, und als Verbraucher sind sie vielfach nicht in der Lage, ihre Konsumwünsche gegenüber den ihnen zur Verfügung stehenden finanziellen Mitteln abzuwägen. „Brauche ich alles, was ich mir wünsche?“ „Kann ich mir auch wirklich alles leisten?“

Reflexionen zur Sache führen zu der Einsicht, dass die Funktion von Geld an Vertrauen gebunden ist. Die Idee des Geldes lebt davon, dass man es jederzeit gegen Produkte oder Dienstleistungen eintauschen kann. Erst recht ist Sparen, Verleihen und Ausleihen (Kredit) mit Vertrauen verbunden. Man hofft darauf, das Ersparte später noch sinnvoll nutzen zu können. Beim Kredit muss der „Verleiher“ auf die Fähigkeit zur Rückzahlung vertrauen. In der Erfahrungswelt der Kinder taucht diese Vertrauensfrage beim „Ausleihen“ und „Wiederbekommen“ auf.

Nach diesen Überlegungen konkretisieren sich auf der Zielebene, differenziert nach Wissen, Können und Verstehen, folgende Absichten:

- Wissen erarbeiten über: die Herstellung ausgewählter Produkte; den Weg ausgewählter Waren; die Funktionsweise von Geld, Bargeld und 
anderen Zahlungsmitteln; Unterschiede zwischen Bedürfnissen und Wünschen, die Beeinflussung von Kaufentscheidungen, erwünschte und unerwünschte Wirkungen von Produktion und Konsum; Qualitätskriterien für Kaufentscheidungen; Verbraucherrechte, Verbraucherschutz und Verbraucherberatung

- Verstehen entwickeln: Einsicht in das Prinzip der Knappheit; individuelle, soziale und ökologische Folgen des eigenen Konsumverhaltens reflektieren; Risiken und Chancen im Umgang mit Geld erkennen (z. B. Verschuldung, Überschuldung; Verluste); direkte und indirekte Zahlungswege überschauen; Werbung und Information unterscheiden

○ Können fördern: neben den persönlichen auch den sozialen und ökologischen Nutzen wirtschaftlichen Handelns einbeziehen (z. B. fairer Handel; soziales Engagement von Herstellern); wertorientierte KostenNutzen-Betrachtungen bei der Beurteilung eigener Wünsche und Handlungsziele durchführen; sich an Kriterien für nachhaltigen Konsum orientieren; Preise vergleichen; gezielt Produktinformationen einholen, vergleichen und abwägen

Ausgehend von der den Kindern vertrauten Erfahrung, nicht alles erwerben zu können, was man sich wünscht, könnte eine erste thematische Grobgliederung der mehrstündigen Unterrichtseinheit zum Beispiel lauten:

1. Ich habe viele Wünsche - Brauche ich alles, was ich haben möchte?

2. Ein Fahrrad für ein Stück Papier? - Wie „funktioniert" Geld?

3. Vom Gold zum Geld - Zahlungsmittel früher und heute

4. Mein Taschengeld - Woher kommt es, wohin geht es?

5. Heute schon an morgen denken - Sparen (Girokonto, Sparkonto, Zinsen)

6. Leihst du mir mal deinen Stift? - Grundregeln des Ausleihens und Verleihens

7. Bekomme ich das wieder? - Vertrauen als Voraussetzung für das Ausleihen

8. Gibst du mir ..., ich gebe dir dafür ...-(Gerechtes) Tauschen, Vertrauen

9. Ist das gerecht? - Arm und reich

10. So haben viele etwas davon - Fairer Handel

11. Erst nachdenken, dann kaufen - Die Idee der Nachhaltigkeit

Die Unterrichtseinheit ließe sich thematisch auch anders komponieren. Letztlich hängt es von der konkreten Klasse ab, wie man Schwerpunkte setzt und konkret vorgeht. Darum sollen die Überlegungen zur Konkretisierung der Planung auch an dieser Stelle abschließen. Die konkrete Planung kann so vielseitig und unterschiedlich ausfallen, wie das Ensemble an Gewohnheiten, Bedürfnissen, Fähigkeiten und Rahmenbedingungen, die im Unterricht zusammenkommen - und zwar auf Schüler- und Lehrerseite. Planung auf der Basis wissenschaftlichen Wissens und akzeptierbarer Theorien über Lernen und Lernvoraussetzungen sollte diese Unterschiede nicht einebnen. Sie soll sie vielmehr fruchtbar und sinnvolle Möglichkeiten erkennbar machen, aber 
auch Schwierigkeiten und Grenzen. Dies wiederum ist Voraussetzung für eine rationale Kommunikation über die mit einer Unterrichtsplanung implizierten Erwartungen - und Hoffnungen. Vieles ist möglich, ob es auch sinnvoll ist, erweist sich auch mit der Qualität der Begründungen für Planungsentscheidungen.

\section{Literatur}

Egmont Ehapa Verlag (Hrsg.) (2009): Kids-Verbraucheranalyse 2009. Unter: www.ehapa.de (erschienen am 11.8.2009)

Gadamer, H. G. (1967): Kleine Schriften I. Philosophische Hermeneutik. Tübingen: Mohr

Giest, H.; Hartinger, A. \& Kahlert, J. (Hrsg.) (2008): Kompetenzniveaus im Sachunterricht. Bad Heilbrunn: Klinkhardt

Helmke, A. (2009): Unterrichtsqualität und Lehrerprofessionalität. Diagnose, Evaluation und Verbesserung des Unterrichts. Seelze: Kallmeyer

Kahlert, J. (2009): Der Sachunterricht und seine Didaktik, 3. Auflage. Bad Heilbrunn: Klinkhardt

Kiel, E. (2008): Strukturierung. In: Kiel, E. (Hrsg.) (2008): Unterricht sehen, analysieren, gestalten. Bad Heilbrunn: Klinkhardt, 21-35

Luhmann, N. (1997a): Die Gesellschaft der Gesellschaft, Band 1. Frankfurt am Main: Suhrkamp

Luhmann, N. (1997b): Die Gesellschaft der Gesellschaft, Band 2. Frankfurt am Main: Suhrkamp

Merrill, D. (2002): First Principles of Instruction. In: Educational Technology Research and Development, 3, 43-59

Meyer, H. (2004): Was ist guter Unterricht? Berlin: Cornelsen Scriptor

Meyer, M.A. (2008): Unterrichtsplanung aus der Perspektive der Bildungsgangforschung. In: Zeitschrift für Erziehungswissenschaft, Sonderheft 9, 117-137

Oser, F. (2001): Standards: Kompetenzen von Lehrpersonen. In: Oser, F. \& Oelkers, J. (Hrsg.): Die Wirksamkeit der Lehrerbildungssysteme. Zürich: Rüegger, 215-342

Sandfuchs, U. (2006): Grundfragen der Unterrichtsplanung. In: Arnold, K.-H.; Sandfuchs, U. \& Wichmann, J. (Hrsg.): Handbuch Unterricht. Bad Heilbrunn: Klinkhardt, 685-694.

Sekretariat der Ständigen Konferenz der Kultusminister der Länder: Standards für die Lehrerbildung: Bildungswissenschaften. Online auf Homepage: www.kmk.org/fileadmin/veroeffent lichungen_beschluesse/2004/2004_12_16-Standards-Lehrerbildung.pdf .

Simmel, G. (1906/ ${ }^{4}$ 1992): Psychologie der Diskretion. In: Dahme, H.-J. v./Rammstedt, O. (Hrsg.): Schriften zur Soziologie. Eine Auswahl. Frankfurt am Main: Suhrkamp, 151-158

Spranger, E. (1962): Das Gesetz der ungewollten Nebenwirkungen in der Erziehung. Heidelberg.: Quelle \& Meyer

Terhart, E. (2005): Grundschularbeit als Beruf. In: Einsiedler, W. u.a. (Hrsg.): Handbuch Grundschulpädagogik und -didaktik. Bad Heilbrunn: Klinkhardt, 129-140.

Wiater, W. (2006a): Analyse und Beurteilung von Unterricht. In: Arnold, K.-H.; Sandfuchs, U. \& Wichmann, J. (Hrsg.): Handbuch Unterricht. Bad Heilbrunn: Klinkhardt, 701-709

Wiater, W. (2006b): Didaktische Theoriemodelle und Unterrichtsplanung. In: Arnold, K.-H.; Sandfuchs, U. \& Wichmann, J. (Hrsg.): Handbuch Unterricht. Bad Heilbrunn, 675-685 